\title{
EL “CARAVANING” EN EL DECRETO 26/2018, DE ORDENACIÓN DE CAMPAMENTOS DE TURISMO DE ANDALUCÍA
}

\author{
María Asunción Grávalos Gastaminza \\ Universidad de Huelva \\ https://orcid.org/0000-0003-1425-141X \\ Rocío Hernández Garrido \\ Universidad de Huelva \\ https://orcid.org/0000-0001-7527-9809
}

Cinta Pérez Calañas

Universidad de Huelva

https://orcid.org/0000-0003-3169-9531

\section{RESUMEN}

El Decreto 26/2018, de 23 de enero, de ordenación de campamentos de turismo en Andalucía regula el sector del caravaning desde el punto de vista turístico y del libre mercado. Este estudio analiza la percepción empresarial y efectos del Decreto a través de un cuestionario y tablas de frecuencia. Los resultados muestran que el Decreto ha sido positivo para el sector del caravaning en Andalucía a pesar de algunas limitaciones en aspectos que dificultan el acceso de operadores al sector. Además, sirve de base para la elaboración de normativas en otras comunidades que deseen un desarrollo del sector.

Palabras claves: Turismo; caravaning; campamento de turismo; decreto; Andalucía.

The "caravaning" in the Decree 26/2018 on Ordenation of Tourism Camps in Andalusia

\section{ABSTRACT}

The Decree 26/2018, of January 23, of management of tourism camps in Andalusia regulates the caravanning sector from the tourist and free market point of view. This study analyzes the perception of entrepreneurs and effects of the Decree through a questionnaire and a

Fecha de recepción: 10 de junio de 2019.

Fecha de aceptación: 6 de abril de 2020.

Facultad de Ciencias Empresariales y Turismo. Universidad de Huelva. 21002 HUELVA (España). E-mail: gravalos@dem.uhu.es, rocio.hernandez@dem.uhu.es, cintia.calanas@dem.uhu.es 
table of frequencies. The results show that the Decree has been positive for the caravanning sector in Andalusia in spite of some limitations that make harder the access of operators to this sector. Furthermore, it is useful as a basis for the elaboration of regulations in other communities that wish to develop the sector.

Keywords: Tourism; caravanning; tourist camp; decree; Andalusia.

\section{INTRODUCCIÓN}

En la actualidad, el sector turístico posee una gran importancia en Andalucía, siendo esta Comunidad Autónoma un destino líder y muy popular no sólo en España sino internacionalmente, debido principalmente a sus condiciones geográficas y climatológicas (INE, 2020).

De hecho, el turismo es un elemento indispensable para analizar e interpretar la realidad económica, social, territorial y cultural de Andalucía en particular y de España en general (IECA, 2018). Dentro del sector turístico de esta región, es relevante el nacimiento del caravaning, siendo uno de sectores menos afectados por la crisis (El diario, 2018).

El "caravaning" es un anglicismo que procede de las palabras francesa "caravane" y persa "karvan". Se trata de un tipo de turismo independiente que permite moverse con mucha autonomía durante las vacaciones, estando en contacto continuo con el entorno natural y rural y, además, permite experimentar la sensación de libertad (Colom-Larrosa, Albert; Garcia-Guirrado, 2007). En la normativa inglesa y como apunta Guillén (2014) encontramos el término "caravan" definido como "cualquier estructura diseñada o adaptada para la vida humana que es capaz de moverse de un lugar a otro (ya sea remolcado o transportado en un vehículo), además de cualquier vehículo a motor diseñado o adaptado, excluyéndose del concepto el material rodante ferroviario (...) o cualquier tienda de campaña (...) y que además pueda desplazarse por carretera por sus dimensiones."

Este fenómeno del "caravaning" tiene sus raíces a finales del siglo XIX, afianzándose como modalidad de ocio o de vacaciones tras la Segunda Guerra Mundial en países como Reino Unido (Guillén 2014), Francia u Holanda (Melgosa, 2003). En España la primera regulación data del año 1956, con el Decreto del 14 de diciembre. A partir de esta regulación se crean los primeros campings.

El continuo crecimiento del caravaning, ha llevado a la aparición de una nueva modalidad de turismo. Se trata de un perfil de turista que viaja en autocaravana, con unas necesidades de servicios y alojamientos alejadas de la oferta tradicional de campings. Los vehículos que utilizan para viajar y pernoctar les proporcionan independencia, autonomía y movilidad.

Esta nueva modalidad turística, el caravaning, vive en nuestro país un proceso de expansión en cuanto al número de adeptos, a los que se suman autocaravanistas procedentes de toda Europa que eligen nuestro país para sus vacaciones y viajes, generando una demanda de servicios y alojamientos especializados que se plasman en la creación de un nuevo flujo económico y empresarial que tiene por base al caravaning.

Como apunta Melgosa (2002), los principales alicientes para la práctica del caravaning son la vida al aire libre y el contacto con la naturaleza, pero además, como indica Bayón 
(1999) tiene como fundamentos sociales el turismo, la naturaleza, la juventud, la improvisación, la economía, deporte, aventura, paisaje, entre otros. Además, podríamos añadir que, en muchos casos, es una forma de viajar con mayor frecuencia. Su importancia es tal que, por ejemplo, sólo en España, en el año 2019 se matricularon 8.903 autocaravanas, frente a las 7.046 del año 2018. Este incremento supone un 19,2\% en las ventas anuales de este tipo de vehículos (ASEICAR, 2020). Sin embargo, a pesar de su importancia, pocos estudios en la literatura examinan este sector que actualmente se encuentra en auge.

Por lo tanto, la regulación del sector desde el punto de vista turístico y de la oferta y demanda es una necesidad fehaciente.

Aunque el sector de los campamentos de turismo presentan distintas regulaciones desde el Decreto de 14 de diciembre de 1956 del Ministerio de Información y Turismo, estas normas han pasado por distintas etapas y textos (Melgosa, 2003).

Actualmente, todas las Comunidades Autónomas de España, gozan de normativas especificas en lo que a campismo y camping se refiere, y en cierto modo tienen puntos en común entre todas ellas, pero la proliferación de nuevos establecimientos de alojamientos especializados en este sector como las áreas de pernocta para autocaravanas, han llevado a algunas Comunidades Autónomas a articular normativas específicas para esta nueva modalidad alojativa tan en auge.

A diferencia de otros marcos normativos que pueden darse en otros países de nuestro entorno, donde se pueden discernir entre espacios para el alojamiento en caravanas con un carácter de permanencia o residencial y aquellos de carácter vacacional (Guillén, 2015), en España sólo se regulan estos últimos no contemplándose el escenario de tipo residencial por no considerarse instalaciones de alojamiento turístico.

Como punto de referencia a nivel nacional, cabe destacar el caso de la Comunidad de Andalucía, donde dicha regulación se realizó con el Decreto 26/2018, de 23 de enero, de ordenación de los campamentos de turismo en la comunidad Autónoma de Andalucía. El objeto de este Decreto es regular los campamentos de turismo como tipo de establecimiento de alojamiento turístico. Según el Decreto 26/2018, podemos definir los campamentos de turismo como "aquellos establecimientos de alojamiento turístico que, ocupando un espacio de terreno debidamente delimitado, acondicionado y dotado de instalaciones y servicios precisos, se destinen a facilitar a las personas usuarias de servicios turísticos un lugar adecuado para hacer vida al aire libre, a cambio de precio y durante un período de tiempo limitado, sin que en ningún caso la permanencia de las personas turistas en estos establecimientos pueda tener carácter de residencia habitual o de domicilio".

Con anterioridad a la normativa andaluza, se publicaron los Decretos del País Vasco (Decreto 396/2013, de 30 de julio, de ordenación de los campings y otras modalidades de turismo de acampada en la Comunidad Autónoma de Euskadi) y de la Comunidad Valenciana (Decreto 6/2015, de 23 de enero, del Consell, regulador de los campings y de las áreas de pernocta en tránsito para autocaravanas de la Comunidad Valenciana). Si bien estos han sido el marco de referencia hasta ahora, el nuevo Decreto andaluz va más allá con esta nueva modalidad alojativa y debe de ser el nuevo marco de referencia.

Con posterioridad se han publicado dos nuevos decretos que regulan los campamentos de turismo, incluyendo las áreas específicas para autocaravanas, en las comunidades de Cantabria (Decreto 94/2018, de 18 de diciembre, por el que se regula la ordenación de 
los campings y de las áreas para autocaravanas de Castilla-La Mancha). Sin embargo, no lo ha logrado ser en las Comunidades de Cantabria y Castilla la Mancha principalmente por no contar estos territorios aun con un sector empresarial dedicado a las áreas para autocaravanas como si sucede en Andalucía, donde el sector ha hecho valer su peso para poder contar con un marco jurídico en el que desarrollarse.

El nuevo Decreto andaluz regula aquellas instalaciones en las que las autocaravanas pueden acampar, esto es, las áreas de pernocta para autocaravanas, además de a los campings. Ambos tipos suponen un gran revulsivo para este sector, ya que a los primeros se los regula por primera vez, sentando las bases para la creación de nuevos establecimientos; y a los segundos, se les hace estar adaptados a las necesidades tan específicas de este tipo de vehículos y que requieren de unas infraestructuras propias y un dimensionado de los que hasta ahora la mayoría de los campings de la comunidad autónoma carecían. Sin embargo, a pesar de la importancia de este nuevo Decreto, no hay estudios del sector del caravaning y de este nuevo Decreto.

Por ello, el objetivo de esta investigación es estudiar en profundidad este nuevo Decreto desarrollado en Andalucía, analizando sus antecedentes, necesidad, importancia de la participación ciudadana en la elaboración del mismo y la afección a los operadores actuales, ya que puede sentar las bases para una regulación en las demás Comunidades Autónomas a nivel nacional y con ello el despegue de este nuevo sector alojativo con todo su potencial. Además, el estudio explora la percepción de los empresarios del sector ante la aparición del Decreto, exponiendo las vías claves y necesarias para su desarrollo.

\section{ANÁLISIS DEL DECRETO}

Desde enero de 2018, Andalucía ha pasado a formar parte de las comunidades autónomas que regulan mediante decreto a las áreas de autocaravanas como instalación de alojamiento turístico. Con este objetivo se ha aprobado el Decreto 26/2018, de 23 de enero, de ordenación de los campamentos de turismo, y de modificación del Decreto 20/2002, de 29 de enero, de Turismo en el Medio Rural y Turismo Activo. Esta nueva normativa afecta a dos ámbitos turísticos muy diferentes, los campamentos de turismo y el Turismo en el Medio Rural y Turismo Activo. El presente estudio se centra en los Campamentos de Turismo ya que prácticamente todo el Decreto se centra en ellos. Los campamentos de turismo se dividen en dos grupos, las áreas de pernocta para autocaravanas y los campings, ciñéndonos expresamente al sector autocaravanista por su gran repercusión y auge en esta comunidad autónoma.

La doble característica de la autocaravana como vehículo o como elemento de acampada siempre ha hecho de él un sector confuso de regular y era necesario establecer unas definiciones claras para una correcta ordenación del mismo. De esta forma, el Decreto andaluz establece que una autocaravana está acampada cuando despliega elementos en su exterior como toldo, mesa o sillas; y, por el contrario, se encuentra estacionada cuando, estando detenida, las condiciones en las que se encuentra son las mismas que circulando. En cuanto a las normas relativas a la circulación, en el régimen de estacionamiento le son aplicables las de los vehículos y, en el régimen de acampada, las relativas a campamentos de turismo. 
Además, este Decreto excluye expresamente las áreas de estacionamiento habilitadas para dar servicio a las autocaravanas, ya sea en el ámbito urbano, interurbano o cualquier otro regulado por su propia normativa sectorial.

\subsection{Antecedentes legislativos, necesidad y objeto}

El antecedente normativo de referencia para esta modalidad turística es el Decreto 164/2003, de 17 de junio, de ordenación de campamentos de turismo, anterior a la Ley 13/2011, de 23 de diciembre, del Turismo de Andalucía por lo que se hacía necesario adaptar este tipo de alojamientos turísticos a la misma, realizar una revisión del sistema de clasificación enfocado a la modernización de los servicios ofertados y al ajuste de los requisitos estructurales de los campamentos de turismo.

En la práctica, el mercado se estaba adaptando a la nueva demanda de servicios especializados de esta modalidad tan específica de turismo, creándose instalaciones para la acogida y servicio de autocaravanas en un vacío legal que hacía poner en riesgo nuevas inversiones. De esta forma, han ido surgiendo áreas para autocaravanas públicas y privadas, en algunos casos amparadas por diversas ordenanzas municipales y, en otros casos, en un vacío legal evidente, con el consiguiente riesgo empresarial que ello implica. Mientras el sector del camping, amparado en el Decreto 164/2003, pretendía defender su oferta tradicional frente a las nuevas áreas, tanto públicas como privadas, y frente a las pernoctas fuera de establecimientos con la presión ante las administraciones. De esta forma, los campings presionaban a los correspondientes ayuntamientos para que no permitieran la pernocta o estacionamiento en sus términos municipales. Además, también presionaban a la Administración autonómica para que regulara este nuevo sector que tanto estaba creciendo y que, en gran parte, no considera a los campings entre sus opciones de alojamiento.

Por lo tanto, la administración andaluza tenía que regular una situación demandada por cuatro actores diferentes y en conflicto como son las administraciones locales, los campings, las áreas privadas de autocaravanas y los usuarios. La administración local, demandaba un marco jurídico en el que basarse para ordenar el flujo de autocaravanistas que llegan a los municipios y una regla en la que encajar urbanísticamente las nuevas instalaciones de licencia privada. Por su parte, los campings demandaban que se incluyeran a las áreas de autocaravanas como establecimientos turísticos reglados, pero introduciéndoles una serie de características como la limitación a estancias muy cortas o una oferta de servicios e infraestructuras no necesarias, para asegurarse su permanencia y supremacía de su tradicional modelo de negocio. En cuanto a las áreas privadas de autocaravanas, éstas demandaban un marco jurídico en el que desarrollar con garantías y libertad su actividad empresarial, que el uso del espacio exterior de la autocaravana con el despliegue de toldo y uso de mesa y silla fuera posible, que las estancias no estuvieran limitadas y que el nivel de infraestructuras se ajustara a la demanda real de los usuarios. El principal objetivo de los usuarios era que se garantizara el derecho al estacionamiento de las autocaravanas fuera de los establecimientos reglados.

Considerando los objetivos de los ciudadanos afectados y teniendo en consideración que la Junta de Andalucía debía cumplir con lo establecido en el artículo 45.c) de la Ley 6/2006, de 24 de octubre, del Gobierno de la Comunidad Autónoma de Andalucía, que 
regula el procedimiento de elaboración de los reglamentos de la siguiente manera: "Cuando una disposición afecte a los derechos e intereses legítimos de la ciudadanía, se le dará audiencia, durante un plazo razonable y no inferior a quince días hábiles, directamente o a través de las organizaciones y asociaciones reconocidas por la ley que la agrupe o la represente y cuyos fines guarden relación directa con el objeto de la disposición. La decisión sobre el procedimiento escogido para dar audiencia a la ciudadanía afectada será debidamente motivada en el expediente por el órgano que acuerde la apertura del trámite de audiencia”. Se estableció trámite de audiencia a distintas entidades en relación con el proyecto de decreto de ordenación de los campamentos de turismo y de modificación del decreto 20/2002, de 29 de enero, de turismo en el medio rural y turismo activo. Según la resolución, "La apertura de trámite de audiencia abreviado durante un plazo de siete días hábiles, además de a las Consejerías de la Administración de la Junta de Andalucía, a las siguientes organizaciones y asociaciones representativas de derechos e intereses legítimos de la ciudadanía afectada por el mismo, considerando que la pluralidad de interesados hace más adecuado este medio para llevarlo a cabo: Federación Andaluza de Municipios y Provincias (FAMP); Confederación de Empresarios de Andalucía (CEA); Unión General de Trabajadores (UGT); Comisiones Obreras de Andalucía (CC.OO); Federación Andaluza de Consumidores y Amas de Casa (Al-Andalus); Federación de Asociaciones de Consumidores y Usuarios en Acción (FACUA Andalucía); Unión de Consumidores de Andalucía (UCA/UCE); Confederación de Asociaciones Vecinales de Andalucía (CAVA); Ecologistas en Acción - Andalucía; Confederación de Entidades para la Economía Social de Andalucía (CEPES); Asociación Andaluza de Autocaravanistas (ASANDAC); Asociación Española de Autocaravanas y Campers (ASESPA); Asociación Áreas Privadas Autocaravanas (AAPA); Plataforma Autocaravanas Autónomas (La Paca) ”.

Por lo tanto, la Administración autonómica se enfrentaba a una situación en la que tenía que actuar en equilibrio con las competencias que le asisten en materia de turismo en contraposición a las competencias que tienen los ayuntamientos en materia de ordenación y gestión del tráfico en las localidades. Podía ordenar la acampada, pero no el estacionamiento. Por ello, quedan fuera del ámbito de aplicación del Decreto todas aquellas instalaciones de acogida y servicio a las autocaravanas donde se estacione, pero no se acampe.

Ante esta situación, la Junta de Andalucía se marca como objeto de este nuevo Decreto el regular los campamentos de turismo como establecimientos de alojamiento turísticos, clasificándolos en dos grupos; los campings, que comprende cinco categorías clasificadas por estrellas y especialidades de carácter voluntario y las Áreas de pernocta de autocaravanas, de categoría única.

Especialmente relevante es la regulación de las áreas de pernocta de autocaravanas, como establecimiento turístico para la acampada de autocaravanas, con el objetivo de regular y dotar de espacios en los que los autocaravanistas dispongan de los servicios, instalaciones y equipamientos destinados a satisfacer las necesidades propias de las autocaravanas y sus ocupantes. Este nuevo modelo alojativo no debe de confundirse con el concepto de estacionamiento o parking, los cuales quedan fuera del ámbito de aplicación de este Decreto.

Todo este esfuerzo de regulación y ordenación del turismo de autocaravanas en Andalucía por parte de la Consejería de Turismo y Deporte en su ámbito competencial en el sector de los campamentos de turismo se queda incompleto por no poder ordenar el 
estacionamiento en la comunidad autónoma de este tipo de vehículos. Para complementar y llegar a tener una ordenación completa ofrece, a través de la FAMP, el asesoramiento necesario a los municipios para una adecuada ordenación del autocaravanismo que se complemente con la normativa autonómica. De esta forma se publica el día 10 de abril de 2018 una "Ordenanza Municipal Tipo de Autocaravanas, Campers y Caravanas" que modifica a la anterior existente y que se adapta al nuevo Decreto autonómico en la materia. Como bien indica, es una ordenanza tipo que se sugiere a los ayuntamientos, pero éstos la pueden adaptar a las necesidades y problemas particulares que pudieran darse en sus municipios. Así mismo, estas ordenanzas deben conjugarse, al menos, con el Reglamento General de Circulación, aprobado por el Real Decreto 1428/2003, de 21 de noviembre el cual regula los regímenes de parada y estacionamiento, y la Instrucción 08/V-74 de la Dirección General de Tráfico del Ministerio del Interior, la cual recopila aquellos aspectos que desde la normativa sobre tráfico y vehículos a motor afectan a las autocaravanas.

En la actualidad podemos encontrar en Andalucía instalaciones de servicio y pernocta para autocaravanas que se encontrarían definidas en el mismo y otras que estarían fuera de su objeto.

En cuanto aquellas que están bajo el dominio del Decreto en estudio, se encuentran los campings generalistas, los campings especialidad autocaravanas o campers (el decreto permite esta posibilidad, pero aún no hay ninguna instalación adherida a esta especialidad) y las áreas de pernocta para autocaravanas.

\section{Figura 1 \\ FIGURAS CREADA POR EL DECRETO}

\begin{tabular}{|c|}
\hline FIGURAS CREADAS POR EL DECRETO \\
\hline Campings (sin espacialidad, generalista) \\
\hline Campings, especialidad: autocaravana o camper \\
\hline Áreas de Pernocta Autocaravanas \\
\hline
\end{tabular}

Fuente: Elaboración propia.

Designamos como camping generalista aquel que representa la oferta tradicional de camping, los cuales cuentan con oferta de bungalow y de parcelas para la estancia de tiendas de campaña, caravanas y autocaravanas. Aquellos que admitan autocaravanas, deben de cumplir los requisitos establecidos para las áreas de pernocta para autocaravanas.

Un camping especialidad autocaravana dedica el 60\% de su superficie de acampada a las autocaravanas y debe de cumplir con aquellos requisitos establecidos para las áreas de pernocta para autocaravanas. Es una especialidad voluntaria (Decreto 26/2018).

Las áreas de pernocta para autocaravanas son instalaciones destinados a la acogida y acampada de autocaravanas, caravanas o campers, así como a sus usuarios, con el fin de descanso y mantenimiento característico de estos vehículos (Decreto 26/2018).

Respecto aquellas que están fuera de su ámbito de aplicación, podemos encontrar las áreas de estacionamiento para autocaravanas privadas, establecimientos de estacionamientos especializados en autocaravanas, áreas para autocaravanas públicas o estaciona- 
mientos. Además, podemos encontrar puntos de reciclaje, que no es más que un punto de servicio para el suministro de agua limpia y desecho de las aguas grises y negras (wc) en estaciones de servicio o similar donde no se produce el estacionamiento (FAMP, 2018).

\section{Figura 2 \\ OTRAS INSTALACIONES DE SERVICIOS A AUTOCARAVANA}

\begin{tabular}{|c|}
\hline OTRAS INSTALACIONES DE SERVICIOS A AUTOCARAVANAS FUERA DEL ÁMBITO DEL DECRETO \\
\hline Áreas de estacionamiento privadas para autocaravanas \\
\hline Establecimientos de estacionamiento para autocaravanas \\
\hline Áreas para autocaravanas públicas \\
\hline Estacionamientos \\
\hline Puntos de reciclaje \\
\hline
\end{tabular}

Fuente: Elaboración propia.

Las áreas de estacionamiento para autocaravanas privadas son aquellos establecimientos que ofrecen a las autocaravanas un espacio de estacionamiento complementado con una zona de servicio donde suministrarse de agua limpia y vaciar las aguas sucias, principalmente. En estas instalaciones no se acampa.

Los establecimientos de estacionamientos especializados en autocaravanas son aquellos aparcamientos abiertos al público que tienen ciertas plazas de aparcamiento destinadas a autocaravanas, como pueden ser los aparcamientos reservados para este tipo de vehículos en los centros comerciales.

Las áreas para autocaravanas públicas son aquellas instalaciones que habilita una administración pública para dar servicio y estacionamiento a las autocaravanas, pudiendo ser de pago o gratuitas.

Los estacionamientos para autocaravanas son aquellas plazas de aparcamiento que una entidad pública puede reservar exclusivamente para ser ocupadas por autocaravanas.

El trabajo realizado por la Consejería de Turismo para la elaboración del Decreto tuvo su base en multitud de reuniones llevadas a cabo con los distintos agentes implicados por su actividad. De esta forma, desde el inicio estaban claramente identificados los puntos necesarios para regular el autocaravanismo en Andalucía, entre los que se encontraban: asegurar la libertad del autocaravanista en elegir donde puede estacionar; no traspasar el límite competenciales de la Consejería en materia de turismo en detrimento de las competencias municipales en cuanto a la regulación de los estacionamientos; crear un marco normativo que sustentara la actividad turística de las nuevas instalaciones de áreas de autocaravanas privadas surgidas en Andalucía, estableciendo el equipamiento mínimo y régimen de funcionamiento; abrir la puerta a la posibilidad de que haya campings especializados en acoger autocaravanas y mejorar la oferta de estos.

Tal y como puso de manifiesto el Ministerio de Medioambiente de la República de Portugal (2008), el autocaravanista demanda instalaciones especializadas en la acogida de las autocaravanas en los destinos, además de poner en relieve una falta de sintonía entre la oferta actual tradicional de campings y la demanda tan específica del autocaravanista. 
Esto hace que una de las principales recomendaciones para la acogida del autocaravanista en este estudio fuera la creación de áreas para autocaravanas en la región del Algarve.

En Andalucía por similitud al Algarve portugués, debe crear instalaciones específicas para la acogida de las autocaravanas que deben de cumplir una serie de premisas básicas para su aceptación por parte del colectivo autocaravanista y son las siguientes: debe de facilitarse la apertura de toldo e instalar mobiliario en el exterior de la autocaravana con el fin de hacer vida al aire libre; deben ser instalaciones concebidas para satisfacer las necesidades de la autocaravana, con puntos para el vaciado de depósitos de aguas sucias, el suministro de agua potable, poseer parcelas adecuadas en dimensiones y pavimentación para la estancia en autocaravanas, posibilitar la conexión eléctrica para aquellas autocaravanas que lo demanden, deben ser lugares que garanticen la seguridad del usuario y deben de proporcionar accesos y viales suficientes para la circulación de este tipo de vehículos.

Por otra parte, para asegurar la viabilidad empresarial de las áreas de pernocta para autocaravanas es preciso tener en cuenta los siguientes aspectos: exigir un equipamiento adecuado, bien sobredimensionado para satisfacer las necesidades del autocaravanista y de la autocaravana (recordar que una autocaravana es un vehículo equipado como vivienda y como tal dispone de todos los servicios necesarios para sus ocupantes); y no limitar la estancia de los clientes en las instalaciones.

El día 10 de octubre de 2017 la Consejería publica un primer borrador en el que se manifiestan tres puntos clave para las posibilidades de desarrollo del sector de las áreas de autocaravanas en Andalucía:

- Artículo 24.3: "En ningún caso, el periodo de ocupación, en conjunto, será superior de seis meses al año en los campings, ni superior a setenta y dos horas en las áreas de pernocta de autocaravanas".

- Anexo II. 3. c) Tendrá sombra natural o artificial en al menos un 50\%. Los obstáculos (árboles, parasoles, etc.) de sombra estarán señalizados.

- Anexo II. 3.e) Tendrá puntos de toma eléctrica por cada parcela.

La limitación a una estancia máxima de 72 horas para las áreas de pernocta de autocaravanas suponía el mayor problema para este sector ya que esta limitación suponía la inviabilidad económica de este tipo de negocios al estar en la obligación de echar a los clientes a los tres días de haber llegado. Además, suponía un agravio comparativo con los mismos campings, los cuales no tendrían dicha limitación de estancia, salvo la de los seis meses al año con el objetivo de evitar fines residenciales, y con otros competidores del mismo sector en comunidades autónomas vecinas o en Portugal o Marruecos, donde no existe limitación en la duración de la estancia.

Fue en el último informe preceptivo que tuvo que pasar el borrador de Decreto antes de su publicación, el del Consejo Consultivo de Andalucía, el que obligaba a la Consejería a cambiar el texto propuesto.

En este sentido, el Consejo Consultivo de Andalucía (2017) se pronunciaba de esta forma en su Dictamen $n^{\circ} 741 / 2017$ :

Establece el precepto un límite temporal que restringe la actividad a prestar por las empresas que se dediquen a tal actividad. 
(...)

Sin embargo, el Proyecto de Decreto que dictaminamos no contiene ni en su preámbulo ni en su articulado una justificación de la regulación que limita tal actividad, lo que supone una vulneración de la normativa comunitaria y estatal.

En efecto, el preámbulo del Proyecto de Decreto no justifica que se trate de una medida de interés general (...)

De esta forma, el inciso final del precepto "durante un período máximo de estancia de setenta y dos horas" es contrario a la normativa comunitaria y estatal señalada, ya que constituye una limitación injustificada.

Esta observación se hace extensiva al artículo 24.3.

La Agencia de Defensa de la Competencia de Andalucía se pronunciaba es estos términos:

(...) De igual modo, para las áreas de pernocta de autocaravanas se regulan requisitos concretos respecto a los accesos, la iluminación, grifos, punto limpio, sombras, tomas de electricidad, así como el tiempo máximo de pernocta permitido para las autocaravanas (72 horas máximo).

En términos generales, esta regulación, excesivamente prolija y de gran casuística, impone importantes cargas a los operadores económicos para acceder al ejercicio de la actividad. Del análisis de los requisitos y servicios exigidos para la clasificación, cabría plantearse la adecuación de los mismos al principio de proporcionalidad en relación con las razones imperiosas de interés general que justifican la iniciativa normativa. En tal sentido, el cumplimiento del amplio $y$ diverso conjunto de exigencias constituye una afectación a la competencia, e implica un incremento innecesario en los costes, susceptible de acabar convirtiéndose en una barrera de entrada para nuevos operadores, e incluso dificultando el mantenimiento de la equivalencia de los ya instalados como consecuencia del cambio en la regulación.

(...)

En consecuencia, se recomienda un profundo trabajo de revisión de la norma en orden a su flexibilización y simplificación, así como a la disminución del grado de intervención administrativa en aspectos que debieran permanecer en el ámbito de decisión de los operadores económicos, y en cualquier caso, debería de tenerse en cuenta la necesidad de llevar a cabo una homogeneización de los requisitos que deberán cumplir los operadores económicos, con independencia del ámbito geográfico en el que desarrollen su actividad. (...)

Por otra parte el requisito del Anexo II 3.c) sobre el porcentaje de sombras en parcelas del 50\%, efectivamente aprobado en el Decreto, supone un obstáculo difícil de salvar por varios motivos: si se opta por sombra artificial, aparte del impacto paisajístico y visual que las estructuras a montar para la proyección de tal cantidad de sombras supondrían su instalación a un mínimo de 4,5m de altura para no suponer un obstáculo para las autocaravanas y sus accesorios, supondrían una gran inversión económica para las áreas por 
las grandes dimensiones de las mismas. Además, estos toldos limitarían el uso de antenas parabólicas y placas solares de las autocaravanas, con el consiguiente perjuicio para los usuarios. Otro de los motivos por los que es un requisito difícil de cumplir es en el caso de que se opte por sombras naturales. En Andalucía, todas las áreas de pernocta para autocaravanas son de reciente construcción o de nueva implantación, con lo que la plantación de los árboles es muy joven y no llegan aún a dar la cantidad de sombra solicitada en el Decreto, aunque tengan potencial para llegar a cubrir dicho requerimiento. Pero, es más, los árboles tipo que se producen en los viveros ornamentales en España tienen la cruz a una altura de $2,5 \mathrm{~m}$, por lo que ramificarían a esa altura y supondrían un obstáculo para las autocaravanas. Es por ello, que una vez plantados es necesario realizarles una poda de formación para crear una nueva cruz a mayor altura, con lo que estaríamos retrasando entre uno y dos años el desarrollo normal de la copa.

Si por algo se caracteriza el potencial del autocaravanismo en Andalucía es por su gran poder desestacionalizador ya que la temporada de mayor afluencia se da entre los meses de noviembre y abril protagonizado por turistas de origen centro europeo que vienen buscando el sol del invierno. Por lo tanto, la obligación de sombrear el 50\% de las parcelas va en detrimento de la desestacionalización del turismo.

Por otro lado, la exigencia de que todas las parcelas cuenten con toma eléctrica supone dos problemas para el sector teniendo en cuenta que el sistema eléctrico es una de las infraestructuras más costosas de implantar, esto puede ser un factor que impida que entren nuevos competidores al sector. Hay que tener en cuenta que casi todas las autocaravanas cuentan con placas solares que cargan las baterías, con lo que son energéticamente autosuficientes. Actualmente, el suministro de energía a cada parcela, por el volumen de potencia necesaria, es inviable realizarlo con un sistema de autoproducción de energía, como por ejemplo mediante placas solares, con lo que pueden darse casos de proyectos que se quieran emprender en lugares interesantes para el turismo de autocaravanas y que no puedan por ser inviable la conexión a la red eléctrica o no estar disponible en la zona.

\subsection{Inspección y régimen sancionador}

La labor de inspección, comprobación y control recae sobre los servicios de inspección de la Consejería competente en materia de turismo.

Las infracciones que se pudieran cometer en contra de lo dictaminado por el Decreto, incurrirán en responsabilidad administrativa sancionable conforme a la Ley 13/2011, de 23 de diciembre, de Turismo de Andalucía.

\subsection{Período de adaptación}

El Decreto establece, en su Disposición transitoria séptima, que “aquellas áreas o establecimientos que bajo esta misma denominación u otra similar sean preexistentes a la entrada en vigor del Decreto, dispondrán de un plazo de tres años para adaptarse a los requisitos previstos en la norma. Una vez adaptadas, los titulares de la explotación deberán cumplimentar la debida declaración responsable para el ejercicio de la actividad". 


\section{METODOLOGÍA}

El objetivo del presente trabajo es analizar el Decreto y poner en relieve la percepción que de él tiene el sector empresarial, así como las posibilidades de desarrollo que ofrece al sector. Por ello, las construcciones teóricas de esta investigación se determinaron utilizando exclusivamente la estructuración seguida en el Decreto andaluz para asegurar la concordancia del estudio con el mismo debido a la inexistencia de estudios que analicen este Decreto y, en general, el sector del caravaning y su adaptación al mismo. Además, fue necesario llevar a cabo entrevistas personales con profesionales y entidades que participaron en la elaboración del Decreto para poder realizar el estudio del mismo.

A la hora de identificar las variables del estudio, se pretendió garantizar la validez del contenido de los instrumentos de medición en todo momento. De esta manera, se obtuvieron un total de 4 elementos. Así, un primer bloque de preguntas está centrado en la justificación del Decreto. El segundo bloque de estudio se centra en los campamentos de turismo en términos globales. La tercera parte está enfocada hacia la adaptabilidad de los campings tradicionales al sector del caravaning. La última parte está dedicada a la nueva figura de las áreas de pernocta para autocaravanas.

Las preguntas formuladas en el cuestionario están basadas en las principales novedades aportadas en este Decreto con respecto a su equivalente anterior y, específicamente, orientadas al conocimiento del sector del caravaning y a la aparición de las áreas de pernocta para autocaravanas. Además, hemos querido incidir en las cuestiones más críticas planteadas tanto por la Agencia de Defensa de la Competencia y el Consejo Consultivo de Andalucía en la fase de elaboración del Decreto.

En la Tabla 1, presentamos la lista completa de variables que se midieron mediante escalas de múltiples elementos. Se puede ver que la variable "Decreto" que comprende cinco elementos, el concepto "campamentos de turismo" que se estudia mediante once elementos, el concepto "campings" que se mide mediante cinco elementos y el concepto "áreas de pernocta" que comprende siete ítems. Las respuestas de los participantes de la encuesta a cada uno de los ítems de estudio se midieron en una escala Likert de siete puntos, que van desde 1 (= "muy desacuerdo") a 7 (= "muy de acuerdo"). Esta es la forma habitual de medir variables que no son directamente cuantificables u observadas (Iacobucci y Churchill, 2010).

Desde diciembre 2018 a febrero 2019, el cuestionario se administró a las13áreas de autocaravanas existentes en Andalucía que pertenecen a la Asociación de Áreas de Autocaravana de Andalucía. Para ello, se les solicitó participar en el estudio mediante un enlace al cuestionario en línea. El cuestionario se anunció a través de pantallas de bienvenida que contenían el objetivo de esta investigación y dos preguntas de filtrado. También se incluyó una declaración sobre la confianza de las respuestas del usuario en la primera pantalla de bienvenida. A través de la pregunta de filtrado inicial, los responsables de cada área que ya habían completado el cuestionario no pudieron responderlo nuevamente. A los que respondieron que todavía no habían tomado el cuestionario se les dio la segunda pregunta de filtrado, que preguntaba si tenían conocimiento del Decreto. Si tenían conocimiento del Decreto, se les pedía que respondieran el cuestionario. Los sujetos que completaron el cuestionario fueron el 84,61\% del total de áreas de Andalucía. 


\section{Tabla 1 \\ ITEMS MEDIDOS}

\begin{tabular}{|c|c|}
\hline Variables & Items \\
\hline \multirow{5}{*}{ Decreto (D) } & $\begin{array}{l}\text { D1. Considero que era necesaria una regulación del sector de las áreas } \\
\text { para autocaravanas. }\end{array}$ \\
\hline & $\begin{array}{l}\text { D2. En términos generales, la regulación efectuada del sector de las } \\
\text { áreas para autocaravanas ha sido positiva }\end{array}$ \\
\hline & $\begin{array}{l}\text { D3. Considero positivo para el autocaravanismo que los } \\
\text { estacionamientos queden fuera de la regulación }\end{array}$ \\
\hline & $\begin{array}{l}\text { D4. Considero positivo que las áreas para autocaravanas municipales } \\
\text { queden fuera de la regulación }\end{array}$ \\
\hline & $\begin{array}{l}\text { D5. Considero positivo para el sector que se prohíban las } \\
\text { acampadas y pernoctas con fines vacacionales o de ocio fuera de los } \\
\text { campamentos de turismo }\end{array}$ \\
\hline \multirow{11}{*}{$\begin{array}{l}\text { Campamento de Turismo } \\
\text { (CT) }\end{array}$} & $\begin{array}{l}\text { CT 1. Es importante que en las áreas de pernocta para autocaravanas } \\
\text { se pueda acampar. }\end{array}$ \\
\hline & $\begin{array}{l}\text { CT 2. Considero que para las áreas de pernocta para autocaravanas } \\
\text { tiene que existir una categoría única. }\end{array}$ \\
\hline & $\begin{array}{l}\text { CT3. Considero que las modalidades de campamentos de turismo, en } \\
\text { relación con su ubicación (playa, rural, ciudad o carretera) se adaptan } \\
\text { a los criterios de su establecimiento. }\end{array}$ \\
\hline & $\begin{array}{l}\text { CT4. Considero idóneo como signo distintivo de un establecimiento, } \\
\text { la placa normalizada para identificar a las áreas de pernocta para } \\
\text { autocaravanas. }\end{array}$ \\
\hline & $\begin{array}{l}\text { CT5. Considero importante o necesario el requisito de equipar el vial } \\
\text { principal de acceso con las señales de velocidad máxima } 10 \mathrm{~km} / \mathrm{h} \text {, } \\
\text { advertencias acústicas prohibidas y circulación prohibida en horas de } \\
\text { descanso. }\end{array}$ \\
\hline & $\begin{array}{l}\text { CT6. Considero importante que todas las parcelas de las áreas de } \\
\text { pernocta tengan que disponer de suministro eléctrico. }\end{array}$ \\
\hline & $\begin{array}{l}\text { CT7. Considero importante que las áreas de pernocta tengan que contar } \\
\text { con servicios higiénicos. }\end{array}$ \\
\hline & $\begin{array}{l}\text { CT8. Considero idóneo que las áreas de pernocta sean compatibles con } \\
\text { campings cuando sean explotadas por el mismo titular. }\end{array}$ \\
\hline & $\begin{array}{l}\text { CT9. Considero idóneo que aquellos campings que admitan } \\
\text { autocaravanas tengan que cumplir con los requisitos específicos de las } \\
\text { áreas de pernocta en lo relativo a zona muelle y puntos limpios y zonas } \\
\text { de parcelas. }\end{array}$ \\
\hline & $\begin{array}{l}\text { CT10. Considero correcto y ágil que los usuarios deban cumplimentar } \\
\text { y firmar el documento de admisión y el parte de entrada de personas } \\
\text { viajeras en el momento de su recepción. }\end{array}$ \\
\hline & $\begin{array}{l}\text { CT11. Considero suficiente el periodo de ocupación de seis meses al } \\
\text { año. }\end{array}$ \\
\hline
\end{tabular}




\begin{tabular}{ll}
\hline Variables & Items \\
\hline C1. Considero suficiente la adaptación propuesta en el Decreto de los \\
campings para la acogida de autocaravanas \\
\hline C2. Considero ventajoso para el sector del caravaning en Andalucía \\
\begin{tabular}{l} 
la compatibilidad entre el grupo Campings y el de Áreas de pernocta. \\
\hline C3. Considero interesante para el sector del caravaning en Andalucía \\
que las áreas de pernocta para autocaravanas fueran compatibles con \\
otros tipos de alojamientos e instalaciones turísticas como casas rurales \\
u hoteles además de con los campings.
\end{tabular} \\
$\begin{array}{l}\text { C4. Creo que la aparición del camping especialidad autocaravana es } \\
\text { positiva para el sector del caravaning en Andalucía. }\end{array}$ \\
$\begin{array}{l}\text { C5. Creo que el camping especialidad autocaravana surge como } \\
\text { respuesta a una necesidad del mercado. }\end{array}$ \\
$\begin{array}{l}\text { AP1.Considero positivo para el sector que las áreas de pernocta puedan } \\
\text { admitir caravanas. }\end{array}$ \\
$\begin{array}{l}\text { AP2.Considero suficiente el periodo de adaptación al Decreto para las } \\
\text { instalaciones preexistentes. }\end{array}$ \\
$\begin{array}{l}\text { AP3.Considero adecuado el ratio de una zona muelle por cada cuarenta } \\
\text { parcelas o fracción. }\end{array}$ \\
\hline $\begin{array}{l}\text { AP4. Considero adecuado el ratio de un punto limpio por cada veinte } \\
\text { parcelas o fracción. }\end{array}$ \\
$\begin{array}{l}\text { AP5. Considero positivo el requisito de tener un 50\% de sombra en la } \\
\text { zona de parcelas para el autocaravanismo en Andalucía. }\end{array}$ \\
$\begin{array}{l}\text { AP6. Me resulta accesible cumplir este requisito. } \\
\text { AP7. Considero adecuada la relación de aparatos sanitarios por parcela. }\end{array}$ \\
\hline Áreas de Pernocta (AP)
\end{tabular}

Fuente: Elaboración propia.

\section{ANÁLISIS DE DATOS Y RESULTADOS}

Esta investigación se basa en una tabla de frecuencias en la que hemos calculado el promedio de cada uno de los ítems de estudio para poder conocer la percepción del Decreto de los empresarios de las áreas de autocaravana de Andalucía.

De acuerdo con Batanero (2002), las tablas de frecuencia son una técnica que permite el análisis de la variabilidad, la determinación de posibles relaciones entre variables, el diseño de estudios y la experimentación y toma de decisiones adecuadas en situaciones de incertidumbre. 
Tabla 2

PROMEDIO DE LA OPINIÓN SOBRE EL DECRETO

\begin{tabular}{clr}
\hline Variable & Items & Promedio \\
\hline & D1 & 6,7273 \\
& D2 & 6,3636 \\
Decreto (D) & D3 & 4,9091 \\
& D4 & 2,7273 \\
& D5 & 4,5455 \\
\hline
\end{tabular}

Se preguntó a los participantes del estudio sobre la necesidad del Decreto para la regulación de este sector y, como se puede observar en la tabla 2 , la respuesta fue positiva, añadiendo que los efectos del mismo habían sido positivos para el sector. Sin embargo, los profesionales admitieron no estar de acuerdo con que los estacionamientos y las áreas de autocaravana municipales queden fuera del Decreto. Además, no consideran positiva el hecho de que se prohíban las acampadas y pernoctas con fines vacacionales o de ocio fuera de los campamentos de turismo.

Tabla 3

PROMEDIO DE LA OPINIÓN SOBRE LOS CAMPAMENTOS DE TURISMO

\begin{tabular}{rlr}
\hline Variable & \multicolumn{1}{c}{ Items } & \multicolumn{1}{c}{ Valor } \\
\hline & CT1 & 6 \\
& CT2 & 3,7273 \\
& CT3 & 5,8182 \\
& CT4 & 5,3636 \\
Campamento de Turismo (CT) & CT5 & 4 \\
& CT6 & 3,2727 \\
& CT7 & 4,3636 \\
& CT8 & 3,4545 \\
& CT9 & 6,6364 \\
& CT10 & 5 \\
CT11 & 5,1818 \\
\hline
\end{tabular}

En general, se les preguntó a los empresarios sobre el encuadre específico de las áreas de pernocta. La tabla 3 muestra que resultó muy relevante la importancia que le da el sector a la posibilidad de que se pueda acampar en las áreas de pernocta. También, se desprende de la tabla 3 que el sector cree interesante que hubiera distintas categorías para las áreas de pernocta, que la placa distintiva para estos establecimientos se considera correcta y que las modalidades disponibles de ajustan a la realidad del sector. Sin embargo, no se considera de 
importancia que el vial de acceso tenga que estar equipado con las señales reglamentarias de tráfico, que todas las parcelas deban de disponer de suministro eléctrico o que las áreas deban de disponer de servicios higiénicos. Por otra parte, no es relevante para estos empresarios que el grupo de las áreas de pernocta de autocaravanas sea compatible con el grupo de los campings, mientras que consideran bastante relevante que aquellos campings que admitan autocaravanas deban de estar adaptados a ello. Además, consideran correctos el periodo de estancia máxima de 6 meses al año y los procedimientos de entrada y admisión.

\section{Tabla 4 \\ PROMEDIO DE LA OPINIÓN SOBRE LOS CAMPINGS}

\begin{tabular}{|c|c|c|}
\hline Variable & Items & Valor \\
\hline \multirow{5}{*}{ Campings (C) } & $\mathrm{C} 1$ & 4,0909 \\
\hline & $\mathrm{C} 2$ & 5,4545 \\
\hline & $\mathrm{C} 3$ & 4,7273 \\
\hline & $\mathrm{C} 4$ & 3,6364 \\
\hline & $\mathrm{C} 5$ & 3,1818 \\
\hline
\end{tabular}

Con respecto al apartado dedicado a los campings, como podemos ver en la tabla 4 , los empresarios de las áreas no consideran suficiente la adaptación propuesta por el Decreto para la acogida en estos campamentos de turismo de las autocaravanas, aunque avalan con suficiencia que haya compatibilidad entre ambos grupos de instalaciones. Por el contrario, no consideran tan necesario que esta compatibilidad se produjera con otro tipo de establecimientos turísticos. Sobre la aparición del camping especialidad autocaravanas, el sector cree que ni es positiva ni responde a una necesidad del mercado.

Tabla 5

PROMEDIO DE LA OPINIÓN SOBRE LAS ÁREAS DE PERNOCTA

\begin{tabular}{clr}
\hline Variable & Items & \multicolumn{1}{c}{ Valor } \\
\hline & AP1 & 5,2727 \\
& AP2 & 4,7273 \\
& AP3 & 4,5455 \\
Áreas de Pernocta (AP) & AP4 & 3,9091 \\
& AP5 & 2 \\
& AP6 & 2,4545 \\
\cline { 2 - 3 } & AP7 & 3 \\
\hline
\end{tabular}

En cuanto a la opinión de los empresarios de las áreas de pernocta sobre los requisitos específicos que les afecta, según nos muestran los valores de la tabla 5, la posibilidad de poder admitir caravanas les parece positiva; pero, sin embargo, les parece suficiente el periodo de adaptación o les parece excesiva e innecesaria las ratios establecidas en cuanto 
al nivel de infraestructuras y equipamientos requeridos. Sin duda alguna, el requisito de tener sombras en parcelas del $50 \%$ se ve como contraproducente para el desarrollo del sector y de muy difícil cumplimiento.

\section{CONCLUSIONES}

Desde un punto de vista empresarial, el mayor logro de este Decreto es dotar al sector de las áreas de autocaravanas de un marco jurídico en el que desarrollar con seguridad su actividad empresarial. Aunque, tal y como indican los empresarios del sector es mejorable, sobre todo en algunos aspectos que dificultan el acceso de nuevos operadores al mercado como son la gran cantidad de sombras establecida para la zona de parcelas o la obligatoriedad de las conexiones eléctricas para todas las parcelas. Esto permite el desarrollo de la actividad empresarial al no haberse publicado con la restricción en la estancia a un máximo de 72 horas como se proponía inicialmente en los distintos borradores del Decreto.

Desde el punto de vista del usuario de autocaravanas, se consigue que se identifique de forma clara y concisa cuando una autocaravana se encuentra estacionada y cuando se encuentra acampada, estableciéndose la presente norma sólo para el caso de que se produzca acampada. Además, se establecen las condiciones de ubicación y los requisitos que deben de cumplir las áreas de pernocta para autocaravanas para la seguridad del usuario.

También, el Decreto atiende a otra de las demandas más frecuentes de los usuarios, contemplando que los campings que admitan autocaravanas tienen que estar adaptados a la acogida de este tipo de vehículos y a la prestación de los servicios que demandan, creando incluso una especialidad de carácter voluntario para aquellos establecimientos que así lo deseen.

Por el contrario, el Decreto andaluz no ha sido capaz de regular los espacios habilitados y dotados de equipamientos por los ayuntamientos para la acogida de las autocaravanas, creando una clara desventaja competitiva frente al sector regulado en el Decreto en cuanto a requisitos de instalaciones, equipamiento y gestión con respecto a las promovidas por estos organismos públicos.

Considerando todos estos factores, podemos concluir que esta norma andaluza ha sido capaz de regular este sector de una forma adecuada gracias a la participación de las personas en la elaboración del mismo. Además, en general, la percepción de los empresarios sobre el mismo es positiva.

Para finalizar y atendiendo a los datos arrojados por el empresariado andaluz del caravaning, podemos afirmar que el modelo expuesto en este Decreto es adecuado y puede servir como referente para la elaboración de otras normas en aquellas comunidades autónomas donde se pretenda fomentar este nuevo pujante sector turístico.

\section{BIBLIOGRAFÍA}

BATANERO, C. (2002): "Los retos de la cultura estadística". Conferencia en las Jornadas Interamericanas de Enseñanza de la Estadística, Buenos Aires. Confederación LatinoAmericana de Sociedades de Estadística. 
BAYÓN,F; ASÍN J.M (1999): “Alojamientos extrahoteleros”, en 50 años del Turismo Español. Un análisis histórico y estructural. Madrid. Centro de estudios Ramón Areces, pp. 927-941.

COLOM, A y GARCÍA, J.M. (2007): Libro Blanco del Camping y Caravaning de España. (Fira de Barcelona, Ed.).

GUILLÉN NAVARRO, N.A. (2014): "El régimen legal de los campamentos de turismo en España: del alojamiento turístico a la residencia habitual de ciudadanos en tiempo de crisis", Estudios Turísticos, n ${ }^{\circ}$ 202, pp. 9-28.

GUILLÉN NAVARRO, N.A. (2015): “Análisis normativo y problemática urbanística de los parques de caravanas y mobilehomes en Inglaterra”, Ciudad y Territorio. Estudios Territoriales, $\mathrm{n}^{\mathrm{o}} 185$, pp. 553-569.

IACOBUCCI, D. y CHURCHILL, G.A. (2010): Marketing research: methodological foundations. South-Western Cengage Learning.

MELGOSA ARCOS, F.J. (2002): "La ordenación de los campamentos de turismo y de las acampadas", en Ordenación y gestión del territorio turístico, Valencia, Tirant lo Blanch, pp. 741-792.

MELGOSA ARCOS, F.J. (2003): "Camping” en la obra colectiva Diccionario de la Administración Local, editado por El Consultor de los Ayuntamientos (Grupo La Ley).

MINISTERÍO DO AMBIENTE, DO ORDENAMENTO DO TERRITÓRIO E DO DESENVOLVIMIENTO REGIONAL. (2008): Caracterização do Auto-caravanismo na Região do Algarve e proposta para definição de una estratégia de acolhimento. Recuperado dehttps://www.ccdr-alg.pt/site/sites/ccdr-alg.pt/files/DesenvRegional/ Estudos/auto_caravanismo1.pdf

\subsection{Otras referencias de prensa}

Asociación Española del Comercio e Industria del Caravaning (ASEICAR) (2020). https:// aseicar.org/el-sector-del-caravaning-se-consolida-en-espana-25-matriculaciones-diarias-en-2019 Recuperado 06 de Marzo 2019.

El diario. (2018). El boom del sector del caravaning en España.https://www.eldiario. es/economia/boom-sector-caravaning-Espana_0_805269602.htmlRecuperado $06 \mathrm{de}$ Marzo de 2019.

El País. (2018). Cinco aplicaciones para planificar unas vacaciones en autocaravana | Tecnología | EL PAÍS. https://elpais.com/tecnologia/2018/07/12/actualidad/1531419623_869030.htmlRecuperado el 6 de marzo de 2019.

\subsection{Otras referencias normativas}

Agencia de Defensa de la Competencia de Andalucía. (2017). Informe Nº16/2017, sobre el proyecto de decreto de ordenación de los campamentos de turismo y de la modificación del decreto 20/2002, de 29 de enero, de turismo en el medio rural y turismo activo. http://www.juntadeandalucia.es/defensacompetencia/sites/all/themes/competencia/ files/pdfs/Informe\%20N\%2016-2017.pdf Recuperado 02 de Abril de 2020. 
Agencia de Defensa de la Competencia de Andalucía. (2017). Respuestas a los escritos de alegaciones sobre la regulación de las áreas de pernocta de autocaravanas contenida en el proyecto de decreto de ordenación de los campamentos de turismo y de la modificación del Decreto 20/2002, de 29 de enero, de Turismo en el Medio Rural y Turismo Activo, presentados por la A.A.P.A. http://www.juntadeandalucia. es/defensacompetencia/sites/all/themes/competencia/files/pdfs/Nota\%20sobre $\% 20$ consulta\%20C\%20362017\%20AAPA\%20Proyecto\%20D\%20\%20Campamentos\%20 web.pdf. Recuperado 06 de Marzo 2019.

Consejería de Turismo y Deporte de la Junta de Andalucía. (2017). Borrador 12/04/2017 Proyecto de Decreto XXXXX, de ordenación de los campamentos de turismo y modificación del Decreto 20/2002, de 29 de enero, de Turismo en el Medio Rural y Turismo Activo, de: https://www.juntadeandalucia.es/export/drupaljda/normativa_en_ elaboracion/17/06/PRIMER\%20BORRADOR\%20CAMPAMENTOS\%2012.4.17\%20 def.pdf. Recuperado 16 de enero 2019.

Consejería de Turismo y Deporte de la Junta de Andalucía. (2017). Resolución por la que se establece trámite de audiencia a distintas entidades en relación con el proyecto de decreto de ordenación de los campamentos de turismo y de modificación del decreto 20/2002, de 29 de enero, de turismo en el medio rural y turismo activo. https://www. juntadeandalucia.es/export/drupaljda/normativa_en_elaboracion/17/06/170418\%20 Resol.\%20Tr\%C3\%A1mite\%20Audiencia.pdf Recuperado el 06 de Marzo de 2019.

Consejo Consultivo de Andalucía (2017).Dictamen No741/2017 de 21 de Diciembre. https://server.knosys.es/ccandalucia/knpag?x=index Recuperado 2 de Abril 2020.

Decreto 20/2002, de 29 de enero, de Turismo en el Medio Rural y Turismo Activo. Boletín Oficial de la Junta de Andalucía, Andalucía, 29 de enero de 2002.

Decreto 164/2003, de 17 de junio, de Ordenación de Campamentos de Turismo. Boletín Oficial de la Junta de Andalucía, Andalucía, 17 de junio de 2003.

Decreto 396/2013, de 30 de julio, de ordenación de los campings y otras modalidades de turismo de acampada en la Comunidad Autónoma de Euskadi. Boletín Oficial del País Vasco, País Vasco, 6 de septiembre de 2013.

Decreto 6/2015, de 23 de enero, del Consell, regulador de los campings y de las áreas de pernocta en tránsito para autocaravanas de la Comunidad Valenciana. Diario Oficial de la Comunitat Valenciana, Comunidad Valenciana, 27 de enero de 2015.

Decreto 26/2018, de 23 de enero, de ordenación de los campamentos de turismo, y de modificación del Decreto 20/2002, de 29 de enero, de Turismo en el Medio Rural y Turismo Activo. Boletín Oficial de la Junta de Andalucía, Andalucía, 7 de febrero de 2018.

Decreto 94/2018, de 18 de diciembre, por el que se regula la ordenación de los campings y de las áreas para autocaravanas de Castilla-La Mancha. Diario Oficial de Castilla La-Mancha, Castilla La-Mancha, 26 de diciembre de 2018.

Decreto 51/2019, de 4 de abril, de Ordenación de los Campamentos de Turismo y Áreas de Servicio para Autocaravanas en el Ámbito de la Comunidad Autónoma de Cantabria. Boletín Oficial de Cantabria, Cantabria, 12 de abril de 2019. 
Directiva 2006/123/CE del Parlamento Europeo y del Consejo de 12 de diciembre de 2006 relativo a los servicios en el mercado interior. Diario Oficial de la Unión Europea, Bruselas, 27 de diciembre de 2006.

Federación Andaluza de Municipios y Provincias (FAMP). Ordenanza municipal tipo de autocaravanas, campers y caravanas, 2018.

Instituto de Estadística y Cartografía de Andalucía (IECA). (2018).. Encuesta de Coyuntura Turística de Andalucía. http://www.juntadeandalucia.es/institutodeestadisticaycartografia/iea/consultasActividad.jsp?CodOper=46\&sub=57941Recuperado el 6 de marzo, 2019.

Instituto Nacional de Estadística (I.N.E.). (2020). Número de turistas según comunidad autónoma de destino principal (10823). http://www.ine.es/jaxiT3/Tabla. htm?t=10823Recuperado el 6 de marzo, 2019.

Instrucción 08/V-74. Dirección General de Tráfico del Ministerio del Interior. http://www. aseicar.org/wp-content/uploads/2017/03/dgt.pdf Recuperado 02 de abril de 2020.

Ley 6/2006, de 24 de octubre, del Gobierno de la Comunidad Autónoma de Andalucía. Boletín Oficial de la Junta de Andalucía, Andalucía, 7 de noviembre de 2006.

Ley 17/2009, de 23 de noviembre, sobre el libre acceso a las actividades de servicios y su ejercicio. Boletín Oficial del Estado, 23 de noviembre de 2009.

Ley 13/2011, de 23 de diciembre, del Turismo de Andalucía. Boletín Oficial de la Junta de Andalucía, Andalucía, 23 de diciembre de 2011.

Ley 40/2015, de 1 de octubre de Régimen Jurídico del Sector Público. Boletín Oficial del Estado 12 de octubre de 2015.

Real Decreto 1428/2003, de 21 de noviembre, por el que se aprueba el Reglamento General de Circulación para la aplicación y desarrollo del texto articulado de la Ley sobre tráfico, circulación de vehículos a motor y seguridad vial, aprobado por el Real Decreto Legislativo 339/1990, de 2 de marzo. BOE-A-2003-23514. 